\title{
Bildung für Binnenvertriebene? Über die (statistische) Vernachlässigung einer bedeutenden Gruppe
}

\begin{abstract}
Zusammenfassung
Bewaffnete Konflikte führen zu Vertreibung einer großen Anzahl von Menschen. Die Sicherstellung von Bildungsmöglichkeiten in humanitären Notsituationen und für flüchtende Menschen hat in den letzten Jahren große Aufmerksamkeit erfahren. Dabei wurde eine spezifische Gruppe vernachlässigt: Binnenvertriebene. Dieser Artikel zeigt, dass diese mangelnde Berücksichtigung problematisch ist, da Binnenvertriebene die größte Anzahl aller Vertriebenen darstellen. Der Artikel diskutiert zuerst die negativen Auswirkungen von Vertreibung auf Bildungsangebote und -möglichkeiten und erläutert, warum die schnelle Bereitstellung von Bildungsmöglichkeiten für Binnenvertriebene wichtig ist. Ein Mangel an Daten steht dem jedoch im Weg. Der Artikel erläutert, warum grundlegende Daten zu Binnenvertriebenen für die Versorgung mit Bildungsangeboten in humanitären Notsituationen relevant sind. Abschließend analysiert er verschiedene Gründe für die unzureichende Datenlage. Mit dem Artikel ist auch das Ziel verbunden, das Forschungsfeld Bildung in Notsituationen stärker in den Fokus deutschsprachiger Diskussionen zu bringen. Die Auswirkungen der Covid-19-Pandemie auf Bildungssysteme unterstreichen die Wichtigkeit dieses Forschungsfeldes.
\end{abstract}

Schlüsselworte: Binnenvertriebene, humanitäre Notsituation

\footnotetext{
Abstract

Armed conflicts cause massive displacements. Providing education in humanitarian emergencies and to refugees has received significant attention over the last years. One particular group, however, has largely been neglected: Internally Displaced Persons (IDPs). This article argues that this neglect is problematic, as IDPs make up the largest share of all forcibly displaced persons. To do so, it first discusses the negative impact of armed conflict on education for IDPs and explains why it is important to rapidly restore the provision of education. Data on IPDs, however, are scarce. The article demonstrates the importance of data for providing education for IDPs. Finally, it analyses different reasons for the statistical invisibility of IDPs. The article
}

seeks to contribute to further integrating the field of education in emergencies into Germanophone discussions. The impact of the Covid-19-pandemic highlights the importance of this field of study.

Keywords: Internally Displaced Persons, humanitarian emergency

\section{Einleitung}

Bewaffnete Konflikte, Naturkatastrophen und Epidemien stellen Bildungssysteme vor große Herausforderungen (GCPEA, 2018; Lange, Timm, \& Höck, 2017; UNESCO, 2018). Die Versorgung mit Bildungsangeboten in humanitären Notsituationen und damit jeweils verbundene positive und negative Effekte auf Konflikte und Friedenssicherung, beschäftigen zunehmend die Forschung und Praxis (Bush \& Saltarelli, 2000; Hanf, 2007; Machel, 1996; Novelli, Higgins, Ugur, \& Valiente, 2013; Scheunpflug \& Wenz, 2018; Seitz, 2004). Scheunpflug und Wenz (2018, S. 317) fassen diesen Sachverhalt folgendermaßen zusammen: „Bildung impliziert ein großes Potenzial zur Friedensförderung, kann aber auch dann, wenn durch Bildungsmaßnahmen bestehende gesellschaftliche Ungleichheit verstärkt wird, bestehende Konflikte verschärfen." Der Bildungsbegriff, das sei vorneweg angemerkt, ist selbstverständlich hochgradig komplex und vielgestaltig und seine angemessene konkrete Ausformulierung ist vom Kontext und nationalen Curricula abhängig. Im Folgenden wird unter Bildung vor allem schulische Grundbildung, im Sinne von Basiskompetenzen und entsprechenden Literacy-Konzepten, verstanden. Darüber hinaus spielen im Kontext der Debatte über Bildung in humanitären Notsituationen auch stärker domänenspezifisch orientierte Formen der Bildung, etwa berufliche Bildung, eine Rolle. Oftmals werden Lehrkräfte in grundlegender psychologischer Ersthilfe, dem „gewaltfreien Umgang [...] mit Konflikten ", konfliktsensitiven Bildungsansätzen und Friedenserziehung weitergebildet (Jäger, 2016, para. 1) um die durch Vertreibung ausgelösten Erfahrungen der Schüler/-innen auffangen zu können. Dabei gilt es Formen der Beschulung in einem sicheren Rahmen zu gewährleisten, selbst dann, wenn 
der soziopolitische Kontext hochgradig unsicher ist. Neben diesen inhaltlichen Komponenten fokussieren konfliktsensitive Bildungsprogramme (idealerweise) strukturelle Elemente, wie etwa die Sicherstellung gleicher Zugangschancen zu Bildungseinrichtungen für verschiedene soziale, ethnische oder religiöse Gruppen (Jäger, 2016).

Vor kurzem wurde der Zusammenhang von Bildung und Vertreibung durch den UNESCO Global Education Monitoring Report in den Fokus der internationalen Aufmerksamkeit gerückt: „Migration, Flucht und Bildung: Brücken bauen statt Mauern" (UNESCO, 2018). Vor allem nationale Grenzen überschreitende flüchtende Menschen werden dabei hervorgehoben. Jedoch gibt es eine Gruppe, die bis dato größtenteils vernachlässigt wurde: Binnenvertriebene, von der UN definiert als „ZivilistInnen, die innerhalb ihres Landes auf der Flucht vor Konflikten, Gewalt oder allgemeinen Menschenrechtsverletzungen sind" (UNHCR, n. d.). Nur wenige Publikationen widmen sich den Bildungschancen dieser Gruppe (z. B. Bengtsson \& Naylor, 2016; Brandt, 2019; Ferris \& Winthrop, 2010; Ghaffar-Kucher, 2019; Mendenhall, Gomez, \& Varni, 2018; Shanks, 2019; Smith Ellison \& Smith, 2013; Sommers, 2005).

Alle genannten Publikationen weisen auf die Marginalisierung Binnenvertriebener in nationalen und internationalen Bildungsprojekten hin. Ein grundlegender Faktor ist der Mangel an Informationen über Binnenvertriebene. Der UNESCO Global Monitoring Report 2010 zum Thema „Die unbeachtete Krise: Bewaffneter Konflikt und Bildung" hielt fest, dass die Datenlage zur Bildungssituation Binnenvertriebener noch schlechter ist als die zu geflüchteten Menschen (UNESCO, 2011, S. 158). Daten sind jedoch wichtig für die Erarbeitung effizienter, relevanter und inklusiver Bildungsprojekte. Dieser Artikel baut auf diesen Beobachtungen auf und widmet sich folgenden Forschungsfragen: Warum sind Daten für die Bereitstellung von Bildungsmöglichkeiten für Binnenvertriebene wichtig? Wieso ist diese Datenlage so schlecht? Dafür bietet der Artikel zunächst einen Überblick über das unzureichende Bildungsangebot und die Bedeutung von Bildungschancen für diese heterogene Gruppe. Dann erläutert er die Wichtigkeiten von Daten. In der darauffolgenden Analyse werden Gründe für die schlechte Datenlage diskutiert. Abschließend werden im Fazit Themenfelder und Desiderate für zukünftige Forschung umrissen.

\section{Negative Auswirkungen von Vertreibung auf das Bildungsangebot von Binnenvertriebenen}

Die Auswirkungen von Vertreibung auf Bildungssysteme sind oftmals verheerend. Oft sind bereits marginalisierte und unterprivilegierte Gruppen am schlimmsten von Vertreibung und vom weiteren Verlust von Bildungsmöglichkeiten betroffen (UNESCO, 2011, S. 158). Durch statistische Annäherung schätzt Englund (2018, S. 14), dass mit hoher Wahrscheinlichkeit Kinder und Jugendliche im Schulalter zwischen 29\% und $44 \%$ aller vertriebenen Menschen ausmachen. Ebenso berechnet er, dass zwischen $40 \%$ und $62 \%$ aller binnenvertriebenen Kinder im Schulalter nicht beschult werden. In der Regel verlieren Binnenvertriebene viele Schultage und ggf. Schuljahre (Ferris \& Winthrop, 2010; Ullah, Khan, \& Mahmood, 2017).
Durch die lange Dauer der Vertreibung würden manche Kinder und Jugendliche gar nicht zu Bildungschancen kommen, wenn sie diese nicht während ihrer Vertreibung erhalten (Dryden-Peterson, 2011, S. 3).

Camps bieten eine nur unzureichende Bildungsinfrastruktur und sind oft auf Primärschulbildung beschränkt (Bengtsson \& Naylor, 2016). Einschulungsraten in Grundschulen in Camps variieren sehr stark, zwischen $0 \%$ und 100\% (Dryden-Peterson, 2011). Urbane Räume, in denen ein Großteil der binnenvertriebenen Menschen lebt, stellen große Herausforderungen dar, da gängige Lösungen (z. B. Schulen bauen) dort schwieriger zu ermöglichen sind (Dryden-Peterson, 2011). Darüber hinaus können Binnenvertriebene Zertifizierungen verlieren - Zeugnisse, etc. - und dadurch nur erschwert Zugang zu Bildungseinrichtungen bekommen (Davies, 2013; Kirk, 2009).

Vertreibung kann eine Reihe von Konflikten auch innerhalb von Klassenzimmern und Schulen auslösen: Diskriminierungen jeglicher Art steigen an, wenn grundlegende soziale Normen außer Kraft gesetzt werden und Grundrechte nicht eingefordert werden können (Dryden-Peterson, 2011). Shanks (2019) zeigt z. B. Spannungen in der Autonomane Region Kurdistan auf, verursacht durch das Miteinander von Schüler/-innen verschiedener Ethnien im gleichen Klassenzimmer, die oftmals, zu Recht oder Unrecht, mit unterschiedlichen bewaffneten Gruppierungen assoziiert werden (s. auch Rhoades, 2010). Auch Lehrer/-innen selbst können Vorurteile haben - verstärkt durch die Polarisierung von Gruppenidentitäten in bewaffneten Konflikten - und sind somit nicht zwangsläufig neutrale Begleiter für alle Schüler/-innen (Bengtsson \& Naylor, 2016). Shanks (2019) beschreibt außerdem einen Fall, bei dem binnenvertriebene Lehrer/-innen durch internationale Organisationen besser bezahlt wurden als einheimische Lehrer/-innen durch ihre Regierung. Dies kann zu großen Spannungen führen.

Eine Rückkehr nach der Vertreibung ist oftmals mit weiterem Leid und Gefahren verbunden. Die temporäre und unsachgemäße Nutzung von Schulen durch das Militär, bewaffnete nichtstaatliche Gruppierungen oder andere Binnenvertriebene kann dazu führen, dass rückkehrende Binnenvertriebene abgenutzte und verfallene Schulen auffinden (GCPEA, 2018; O'Malley, 2010, S. 184; Richmond, 2014, S. 25, 185, 190). Brandt (2019) zeigte im Falle der Demokratischen Republik Kongo, dass Regierungen Druck auf binnenvertriebene Lehrer/-innen ausüben können, damit diese in ihre Heimatdörfer zurückkehren - teilweise gegen deren Willen. In einigen Kontexten, z. B. Boko Haram in Nigeria, stellen Schulen, Lehrer/-innen und Schüler/-innen explizite Ziele der bewaffneten Gruppierungen dar, was in manchen Regionen Nigerias zur Zerstörung aller Schulen führte (Higazi, 2016). Währenddessen gibt es viele Gründe, die dafürsprechen, Binnenvertriebenen so schnell wie möglich wieder Zugang zu Bildungseinrichtungen zu ermöglichen.

\section{Warum eine schnelle Bereitstellung von Bildungsmöglichkeiten für Binnenvertriebene wichtig ist}

Die Versorgung mit Bildungsangeboten in humanitären Kontexten bedeutet in den meisten Fällen eine Auswahl an Interventionen aus einem relativ beschränkten Portfolio, wie zum 
Beispiel: Bau von Klassenzimmern und Toiletten; Weiterbildung von Lehrkräften, insbesondere zur psychologischen Ersthilfe, positiven Disziplin und einem aktiven Unterrichtsmodel; Aufholklassen, in denen Inhalte mit schnellerem Tempo durchgenommen werden; Bereitstellung von Schulbüchern und weiteren pädagogischen Materialien; Bezahlung von Schulgebühren und Gehältern. Die Liste könnte fortgeführt werden. Bei der Erarbeitung und Bereitstellung von Bildungsangeboten in humanitären Situationen geht es weniger um Inhalte - abgesehen vom psychosozialen Lernen und der konflikt-sensiblen Friedenserziehung - als um eine schnelle und zielgruppengerechte Bereitstellung, da die Inhalte von den entsprechenden Ländern gesetzt werden. Warum ist eine schnelle Bereitstellung von Bildungsmöglichkeiten für Binnenvertriebene so wichtig?

Der Zugang zu Bildungseinrichtungen kann zum Schutz von Kindern und Jugendlichen beitragen: Rhoades (2010) argumentiert, dass insbesondere Kinder, die nicht zur Schule gehen, von bewaffneten Gruppierungen (zwangs-)rekrutiert werden können. Außerdem kann der Wiederaufbau von Bildungsinfrastruktur eine langfristige und nachhaltige Investition sein und politische Legitimität herrschender Parteien fördern (Dryden-Peterson, 2011) - was in einigen Fällen jedoch auch problematisch sein kann. Viele Binnenvertriebene leiden unter post-traumatischen Belastungsstörungen (Davies, 2013). Schulen können idealerweise eine „safe school“ sein (Moriarty, 2018) und dazu beitragen ein Gefühl der Normalität wiederherzustellen (z. B. Dryden-Peterson, 2011, S. 3; Rhoades, 2010, S. 40) - auch wenn man natürlich in Betracht ziehen muss, dass diese Normalität vor Ausbruch bewaffneter Konflikte in vielen Fällen bereits gewaltvoll gewesen sein kann. Des Weiteren können Lehrer/-innen ebenfalls unter post-traumatischen Belastungsstörungen leiden und ohne weitere Unterstützung nur schwer mit Spannungen im Klassenzimmer umgehen (Mendenhall et al., 2018, S. 23). Jedoch sind Möglichkeiten zur weiterführenden Lehrer/-innen-Ausbildung unter solchen Umständen rar (Mendenhall et al., 2018, S. 13). Ebenfalls wird häufig auf die positiven Effekte von Schulen für die Entwicklung und Stärkung eines Gemeinschaftssinnes und eines positiven Miteinanders der (binnenvertriebenen) Menschen hingewiesen (Gladwell \& Tanner, 2014). Einer der Mindeststandards des International Network for Education in Emergencies (INEE) besagt folgendes: Wenn ein Ort des Lernens für Binnenvertriebene geschaffen wird, kann die Einstellung von Lehrer/-innen und anderem Personal aus der Gastgemeinschaft zu guten Beziehungen beitragen (INEE, 2012, S. 96).

Der Bildungsbegriff im Feld der humanitären Krisen ist auch durch die positiven Auswirkungen von Bildungsangeboten auf andere humanitäre Bereiche zu verstehen (Karamperidou, Richardson, \& Zapata, 2018). Antworten nationaler und internationaler Organisationen auf die Covid-19-Pandemie unterstreichen die Wechselwirkungen des Bildungsbereichs mit anderen humanitären Feldern, vor allem Zugang zu Wasser, Hygiene, körperlicher und psychosozialer Gesundheit und Kinderschutz (Brandt \& Drerup, 2020). Die Bereitstellung von Bildungsmöglichkeiten kann den Zugang zu lebensrettenden Ressourcen und Wissen unterstützen. Außerdem ist bekannt, dass Kinder, die lange nicht zur Schule gehen, mit einer steigenden Wahrscheinlichkeit nicht mehr dorthin zurückkehren. Eine schnelle Wiederherstellung des Zugangs zu Bildungs- einrichtungen führt also auch dazu, dass langfristig Einschulungsraten nicht sinken. Dies ist einer der Gründe, warum die schnelle Wiedereröffnung von Schulen insbesondere in Kontexten, in denen es wenig bis keinen Zugang zum distance learning in Zeiten von Schulschließungen durch Covid-19 oder z. B. Ebola gibt, äußerst wichtig ist. Die Einhaltung von hygienischen und sozialen Normen im Rahmen von Covid-19Desinfizierung, social distancing, etc. - in Binnenvertriebenencamps wird dabei aufgrund der räumlichen Enge jedoch besonders schwierig (ebd.).

\section{Die Wichtigkeit von Daten für die Gewährleistung von Bildungsangeboten}

Ausreichende und zuverlässige Informationen sind ein Grundpfeiler evidenzbasierter Politikgestaltung und Bildungsplanung (Scheunpflug \& Wenz, 2018). Seit vielen Jahren schlägt sich diese Dynamik beispielweise in den PISA-Studien und der finanziellen und technischen Unterstützung von Management-Informationssystemen für Bildungssektoren in Partnerländern wieder. Viele Publikationen weisen jedoch auf die weitgehende Ignorierung und Unsichtbarkeit von Binnenvertriebenen in statistischen Erhebungen hin (Bengtsson \& Naylor, 2016; IDMC, 2019; UNESCO, 2011, S. 158). Mendenhall et al. (2018, S. 8) stellen nebst der schlechten Datenlage, zum Beispiel Einschulungsquoten betreffend, fest, dass so gut wie gar keine Daten über Bedarf, Anzahl und Qualifikationen von Lehrkräften gesammelt werden.

Laut Sommers (2005, S. 213) könnte die Unterrepräsentation von Binnenvertriebenen in Datenerhebungen dazu führen, dass Entscheidungen internationaler Organisationen nicht auf angemessenen Daten aufbauen und dadurch erhebliche Anteile der Bevölkerung vernachlässigen. Rein praktisch bedeutet der Mangel an Daten über Bedarf, Anzahl und Qualifikationen von binnenvertriebenen Lehrer/-innen und Schüler/-innen, dass Projekte nur in sehr geringem Maße bedürfnisorientiert arbeiten können. Tomaševski’s (2001) menschenrechtsbasierter Ansatz bietet eine hilfreiche Unterscheidung zwischen verschiedenen Formen des Zugangs zu Bildungsmöglichkeiten: Availability (Verfügbarkeit), Accessibility (diskriminierungsfreier Zugang), Acceptability (Angemessenheit), und Adaptability (Anpassbarkeit). Ohne ein fundiertes Verständnis der Situation binnenvertriebener Menschen können diese „4As“, und damit das Menschenrecht auf hochwertige Bildung, nicht erbracht werden. Dies beinhaltet Daten über Altersgruppen, Geschlecht, physische und psychische Einschränkungen, ethnische, sprachliche und religiöse Minderheiten und andere wichtige Kriterien. Alles in allem ist eine gute Datenlage eine Bedingung für die Erarbeitung effizienter, inklusiver und relevanter Bildungsprojekte, als auch für deren Evaluierung (Various Organisations, 2016, S. 45).

\section{Gründe für die unzureichende Datenlage über die Bildungssituation Binnenvertriebener}

Im Folgenden diskutiere ich drei Kategorien von Gründen für die unzureichende Datenlage in Bezug auf Binnenvertriebene. Die erste Kategorie bezieht sich auf die Binnenvertriebenen 
selbst, die zweite Kategorie beinhaltet Aspekte auf Ebene der nationalen Verwaltung und die dritte Kategorie beschäftigt sich mit internationalen Akteuren.

Erstens, Binnenvertriebene sind eine heterogene Gruppe mit vielfältigen Erfahrungen und Lebenswegen. Dies macht die Registrierung und statistische Erfassbarkeit schwierig. Die Zahl der Menschen, die durch Konflikte innerhalb ihrer Landesgrenzen vertriebenen wurden, war 2019 mit etwa 45,7 Millionen so hoch wie nie (IDMC, 2020, S. 2). Dazu kommen circa 5,1 Millionen durch Naturkatastrophen Binnenvertriebene - dies ist im Übrigen die erste Schätzung dieser Zahl überhaupt (ebd.). Im gleichen Jahr gab es mit circa 25,9 Millionen Menschen circa halb so viele geflüchtete Menschen wie Binnenvertriebene (UNHCR 2020). Die durchschnittliche Vertreibung Binnenvertriebener dauert mehrere Jahre an. Manche Binnenvertriebene leben in Camps, andere in Städten und wieder andere ziehen sich in die ihre Dörfer umgebende Natur zurück. Die wenigen vorhandenen Daten erfassen größtenteils Binnenvertriebene in Camps, obwohl eine große Anzahl Binnenvertriebener in urbanen Räumen wohnt, wo sie oftmals nicht registriert, schwer aufzufinden und deswegen statistisch besonders stark unterrepräsentiert sind (Englund, 2018, S. 5). Eine andere Gruppe, die eine statistische Erfassung durch ihren Lebensstil erschwert, sind beispielsweise Nomaden (Randall, 2015). Hinzu kommt: Vertreibung und Rückkehr verlaufen nicht linear. Menschen können wiederholt vertrieben werden, und eine Rückkehr bedeutet meist keine Rückkehr in eine sichere Situation; oftmals hat sich an den grundlegenden Problematiken, die zur Vertreibung geführt haben, wenig geändert. Der Großteil der rückkehrenden Binnenvertriebenen kehrt in fragile und prekäre Zustände zurück, wobei viele Menschen zusätzlich mehrmals hintereinander vertrieben werden (IDMC, 2020; UN OCHA, 2018). Der Unterschied zwischen Binnenvertriebenen und flüchtenden Menschen ist fließend, da einige Binnenvertriebene zu flüchtenden Menschen werden können und flüchtende Menschen durch vorschnelle Repatriierung die Binnenvertrieben von morgen werden können (Ghaffar-Kucher, 2019, S. 4). Wobei die statistische Erfassung am Anfang eines Konflikts, einer Naturkatastrophe und der darauf folgenden Vertreibung noch relativ gut gelingt, erschweren die hier aufgezeigten nachfolgenden Etappen dies wesentlich (Various Organisations, 2016, S. 43).

Zweitens sind Dynamiken auf nationaler Ebene zu nennen. Entgegen Scotts (1998) Argument, dass Staaten grundlegende Information über ihre Bevölkerung benötigen, z. B. durch Steuernummern und Personalausweise, gibt es eine Reihe von Gründen warum staatliche Verwaltungen häufig über keine elementaren Details über ihre Bevölkerung verfügen (Brandt, 2018; Kalir \& van Schendel, 2017; Randall, 2016; Sommers, 2005). Zensus, Geburtsregister und Wohnregistrierungen in vielen einkommensschwachen Ländern sind oftmals lückenhaft oder alt. Solche Daten sind zudem oftmals politisch umkämpft, z. B. aufgrund der Abhängigkeit der Anzahl von Abgeordnetenplätzen in nationalen Parlamenten von regionalen Bevölkerungszahlen. Viele lokale Behörden sind gut informiert über die tatsächliche Lage vor Ort (Brandt, 2019; Sommers, 2005, S. 226). Ihr Wissen wird aber selten auf regionaler oder nationaler Ebene gebündelt. Des Weiteren haben sie eine Reihe von Anreizen, Daten fälschlich dazustellen
(Sandefur \& Glassman, 2015). So kann eine überhöht dargestellte Anzahl an Schüler/-innen zu mehr finanzieller Unterstützung führen. Andererseits wird mitunter angeführt, dass Daten über Binnenvertriebene nicht preisgegeben werden können, da diese höchstsensibel sind (Englund, 2018; Various Organisations, 2016). Bewaffnete Konflikte und innerstaatliche Migrationsbewegungen üben weiteren Druck auf bereits überforderte Verwaltungen auf. Außerhalb von Camps sehen Binnenvertriebene keine Notwendigkeit sich neu zu registrieren und werden selten dazu angehalten. Abschließend ist zu sagen, dass die Situation in einigen Ländern mit den meisten Binnenvertriebenen - z. B. Irak/Autonome Region Kurdistan und Demokratische Republik Kongo - durch eine Überlappung verschiedener Anbieter von Schulen, z. B. staatliche und religiöse Organisationen, charakterisiert ist (Brandt, 2018; Shanks, 2019). Eine solche Arbeitsteilung kann dazu führen, dass nationale Ministerien einen nur sehr unzureichenden Überblick über alle Bildungsangebote haben. Selbst wenn es eine relativ zentralisierte Verwaltung gibt, sind Binnenvertriebene in offiziellen Datenerhebungen nicht zwangsläufig als solche zu erkennen. Sie werden zwar mitgezählt, bleiben aber unsichtbar (Various Organisations, 2016). Ein weiteres Problem ist der jährliche Turnus von Humanitarian Response Plans und Management-Informationssystemen für Bildungssektoren. Um effektiv und effizient in humanitären Notsituationen reagieren zu können, brauchen Organisationen und Regierungen Zugriff auf aktuelle Daten (Various Organisations, 2016, S. 45).

Drittens können fehlende Zuständigkeit und Koordination zwischen internationalen Organisationen als Grund für einen Mangel an Wissen über Binnenvertriebene genannt werden. Der Mangel an institutioneller Zuständigkeit jenseits vom Staatsapparat ist ein wichtiger Grund für statistische Marginalisierung. Obwohl normative und praxisorientierte Richtlinien auch zu Binnenvertriebenen Stellung nehmen - z. B. die Guiding Principles on Internal Displacement (UNESCO, 2011, S. 215), die Mindeststandards des International Network for Education in Emergencies (INEE, 2012, S. 111) oder das Guidebook for planning education in emergencies and reconstruction von UNESCO-IIEP (2010) - verhindert der unverbindliche Charakter eine gute Koordination internationaler Organisationen. Im Gegensatz zu flüchtenden oder asylsuchenden Menschen übertreten Binnenvertriebene keine Ländergrenze und unterstehen somit nur indirekt dem Mandat internationaler Hilfswerke wie dem United Nations High Commissioner for Refugees oder der International Organization for Migration. 2006 wurde Bildung zwar Teil des humanitären Cluster Systems der Vereinten Nationen, geleitet von UNICEF und Save the Children, jedoch hat keine humanitäre Organisation ein spezifisches Mandat für Binnenvertriebene. Besonders bei langfristigen Binnenvertriebenen kommt es vor, dass humanitäre Organisationen sich nicht zuständig fühlen. Englund (2018) kritisiert darüber hinaus, dass sich die wenigen vorhandenen Methoden zur Datenerhebung und -präsentation erheblich zwischen verschiedenen Organisationen unterscheiden und damit nur sehr schwierig vergleichbar sind. Die schlechte Datenlage macht wiederum effektive Kooperation noch schwieriger. Der Mangel an Statistiken muss auch im Rahmen der allgemeinen Finanzierungen von Bildungsangeboten in humanitären Krisen gesehen werden. Trotz der steigenden Finanzie- 
rungsströme und diversen Organisationen, die Gelder für Bildungsprojekte in humanitären Notsituationen sammeln oder bereitstellen (z. B. Globale Bildungspartnerschaft, Education Cannot Wait, Katar's Protect Education in Insecurity and Conflict, Dubai Cares, etc.), werden durchschnittlich unter drei Prozent der humanitären Ausgaben in Bildungsprojekte investiert (UN OCHA, 2018, S. 14). Oftmals überwiegt die Notwendigkeit schneller Interventionen über eine langfristigere Verbesserung der Dateninfrastruktur. Außerdem kann das mangelnde Interesse internationaler Akteure durch übergeordnete globale Agenden erklärt werden, vor allem durch die Verquickung von humanitärer Katastrophenhilfe, Entwicklungspolitik und Sicherheitspolitik (Lopes Cardozo \& Novelli, 2018; Novelli, 2010). Gemäß dieser Perspektive stellen Binnenvertriebene keine unmittelbare Gefahr für westliche Länder dar und bekommen deswegen weitaus weniger Aufmerksamkeit und Unterstützung. Demgegenüber steht die normative Perspektive, dass die Erreichung des vierten Ziels für nachhaltige Entwicklung - inklusive, gerechte und hochwertige Bildungsangebote zu gewährleisten und Möglichkeiten des lebenslangen Lernens für alle zu fördern - unmöglich ist, wenn Binnenvertriebene marginalisiert bleiben. Scheinbar ist der Druck durch die nachhaltigen Entwicklungsziele nicht stark genug, um die Bildungsbedürfnisse Binnenvertriebener systematischer zu erfassen.

\section{Fazit und Ausblick}

Dieser Artikel analysiert die Vernachlässigung von Binnenvertriebenen in der Diskussion und im praktischen Umgang mit Bildungsangeboten in humanitären Notsituationen. Er zeigt, dass Binnenvertriebene den Großteil aller weltweit Vertriebenen ausmachen und in einer prekären und komplizierten Bildungs- und Gesamtsituation leben. Angesichts des seit zwanzig Jahren steigenden Interesses am Themenfeld Bildung in humanitären Notsituationen ist es erstaunlich, dass diese große und verwundbare Gruppe verhältnismäßig wenig Aufmerksamkeit bekommen hat.

Der Artikel zeigt insbesondere auf, dass Binnenvertriebene statistisch unterrepräsentiert sind, obwohl Daten über grundlegende Charakteristika - Alter, Geschlecht, Sprache, etc. - die Basis für die Erarbeitung und Evaluierung inklusiver und relevanter Bildungsprojekte sind. Obwohl Foren, Publikationen und Fördertöpfe existieren, die die Verbesserung der statistischen Erfassung binnenvertriebener Menschen zum Ziel haben, hat der Artikel mehrere Gründe aufgezeigt, warum solche Projekte nur schwer umzusetzen sind. In der Tat ist der Mangel an statistischem Wissen über Binnenvertriebene ein Beispiel für die Schwierigkeit, humanitäre Hilfe mit längerfristigen Maßnahmen der Entwicklungszusammenarbeit zu koppeln (Global Education Cluster, 2019, S. 23).

Die Covid-19-Pandemie hat eine der größten Schwierigkeiten, aber vielleicht auch Potenziale, für die Erarbeitung von Bildungsangeboten für binnenvertriebene Menschen aufgezeigt: distance learning. Eine Reihe von Initiativen widmet sich aktuell der Zusammentragung von innovativen technologiebasierten Bildungsangeboten für Vertriebene. ${ }^{1}$ Forschung sollte sich diesem Themenkomplex kritisch widmen und dabei auf folgende beispielhafte Fragen eingehen: Inwieweit können technologiebasierte Angebote helfen, relevante Bildungsprogramme trotz eines Mangels an Daten über Binnenvertriebene anzubieten? Wie verhalten sich technologiebasierte Bildungsangebote für Binnenvertriebene zu bekannten Herausforderungen, Gefahren und Möglichkeiten digitaler Medien (Binder \& Drerup, 2020)? Welche Rolle wird künstliche Intelligenz in der Beschaffung und Verwertung von Daten über die Bildungssituation binnenvertriebener Menschen, als auch in der Erarbeitung und Evaluierung von Bildungsprojekten spielen (UNESCO, 2019)? Eine Analyse dieser Fragen ist wichtig, damit die Inklusion binnenvertriebener Menschen in Bildungsprojekte bestmöglich gelingen kann. Dabei sollte auch „das Zusammenspiel zwischen der unmittelbaren und der strukturellen Friedenspädagogik entschlossener gefördert werden“ (Jäger, 2016, para. 18).

\section{Anmerkungen}

1 Siehe, zum Beispiel, die geplante UNESCO-Studie: „From Radio to Artificial Intelligence: Innovative Literacy Learning and Education for Refugees, Migrants and Internally Displaced Persons“ (UNESCO-ILL, 2020).

\section{Literatur}

Bengtsson, S. \& Naylor, R. (2016). Education for Refugees and IDPs in Low- and Middle-Income Countries. Identifying Challenges and Opportunities. Oxford: HEART (Health and Education Advice and Resource Team).

Binder, U. \& Drerup, J. (2020). Demokratieerziehung und die Bildung digitaler Öffentlichkeiten. In U. Binder \& J. Drerup (Hrsg.), Demokratieerziehung und die Bildung digitaler Öffentlichkeiten (S. 1-11). Wiesbaden: Springer. https://doi.org/10 1007/978-3-658-28169-4

Brandt, C. O. (2018). Illegibility as a State Effect. The Limits of Governing Teacher Identification in the Democratic Republic of Congo. University of Amsterdam, PhD Thesis, unveröffentlicht.

Brandt, C. O. (2019). The Deployment of Internally Displaced Teachers (DR Congo): A 'Real Governance' Approach. International Journal of Educational Development, 66, 147-154. https://doi.org/10.1016/j.ijedudev.2018.10.006

Brandt, C. O. \& Drerup, J. (2020). Bildung in humanitären Krisen: Zu den globalen Folgen von Covid-19. In J. Drerup \& G. Schweiger (Hrsg.), Bildung und Erziehung im Ausnahmezustand. Philosophische Reflexionsangebote zur COVID-19-Pandemie (S. 161-176). Stuttgart: WBG Theiss.

Bush, K. D. \& Saltarelli, D. (2000). The Two Faces of Education in Ethnic Conflict. Towards a Peacebuilding Education for Children. Florenz: UNICEF.

Davies, L. (2013). The Challenges for Education of Protracted Displacement in Sri Lanka. In C. Smith Ellison \& A. Smith (Hrsg.), Education and Internally Displaced Persons (S. 119-141). London: Bloomsbury. https://doi.org/10.5040/97814725 52914.ch-006

Dryden-Peterson, S. (2011). Conflict, Education and Displacement. Conflict and Education, 1(1), 1-5.

Englund, G. (2018). Estimating the number of forcibly displaced school-age children not accessing education. Background paper prepared for the 2019 Global Education Monitoring Report. Paris: UNESCO.

Ferris, E. \& Winthrop, R. (2010). Education and Displacement: Assessing Conditions for Refugees and Internally Displaced Persons affected by Conflict. Background Paper for the Global Monitoring Report 2011. Paris: UNESCO.

GCPEA. (2014). Education Under Attack 2014. New York, NY: Global Coalition to Protect Education from Attack.

GCPEA. (2018). Education Under Attack 2018. New York, NY: Global Coalition to Protect Education from Attack.

Ghaffar-Kucher, A. (2019). Uncertain prospects and ambiguous futures: the challenges and opportunities of educating the forcibly displaced. Background paper prepared for the 2019 Global Education Monitoring Report. Paris: UNESCO.

Gladwell, C. \& Tanner, L. (2014). Hear it from the children. Why education in emergencies is critical. A study on the role of education for conflict-affected communities in the Democratic Republic of Congo and Ethiopia. London: Save the Children UK (prepared together with the Norwegian Refugee Council). 
Global Education Cluster (2019). Strategic Plan 2017-2019 Revision. o. O.: o. V. Hanf, T. (2007). Bildung und Konfliktbearbeitung. Ein bedeutsames Sektorvorhaben der deutschen Entwicklungszusammenarbeit. ZEP - Zeitschrift Für Internationale Bildungsforschung und Entwicklungspädagogik, 30(2), 7-9.

Higazi, A. (2016). IDPs and Education in North-East Nigeria after Boko Haram. Norrag News, 53, 104-105.

IDMC (2019). Twice Invisible. Accounting for internally displaced children. Genf: International Displacement Monitoring Center.

IDMC (2020). Global Report on Internal Displacement. Genf: International Displacement Monitoring Center.

INEE (2012). Minimum Standards for Education: Preparedness, Response, Recovery. New York, NY: Inter-Agency Network for Education in Emergencies.

Kalir, B. \& Schendel, W. van (2017). Introduction: Nonrecording states between legibility and looking away. Focaal-Journal of Global and Historical Anthropology, 77, 1-7. https://doi.org/10.3167/fcl.2017.770101

Karamperidou, D. Richardson, D., \& Zapata, J. (2018). Education in Multi-Sectoral Responses to Displacement Crises. Background paper prepared for the 2019 Global Education Monitoring Report. Paris: UNESCO.

Kirk, J. (Hrsg.) (2009). Certification counts: Recognizing the learning attainments of displaced and refugee students. Paris: UNESCO-IIEP.

Lange, S., Timm, S. \& Höck, S. (2017). Editorial [Bildung und Fluchtmigration]. ZEP - Zeitschrift Für Internationale Bildungsforschung und Entwicklungspädagogik, $40(1), 2$.

Lopes Cardozo, M. T. A. \& Novelli, M. (2018). Education in Emergencies: Tracing the Emergence of a Field. In A. Verger, M. Novelli, \& H. K. Altinyelken (Hrsg.), Global Education Policy and International Development. New Agendas, Issues and Policies (S. 233-254). London: Bloomsbury.

Machel, G. (1996). Impact of armed conflict on children. New York, NY: United Nations \& UNICEF.

Mendenhall, M., Gomez, S. \& Varni, E. (2018). Teaching amidst conflict and displacement: persistent challenges and promising practices for refugee, internally displaced and national teachers. Background paper prepared for the 2019 Global Education Monitoring Report. Paris: UNESCO.

Moriarty, K. (2018). Safe Schools: the Hidden Crisis. A framework for Action to deliver Safe, Non-Violent, Inclusive and Effective Learning Environments. London: Theirworld.

Novelli, M. (2010). The new geopolitics of educational aid: From Cold Wars to Holy Wars? International Journal of Educational Development, 30(5), 453-459. http s://doi.org/10.1016/j.ijedudev.2010.03.012

Novelli, M., Higgins, S., Ugur, M. \& Valiente, O. (2014). The Political Economy of Education Systems in Conflict-Affected Contexts: A Rigorous Literature Review. London: Department for International Development.

O'Malley, B. (2010). Education under Attack. A global study on targeted political and military violence against education staff, students, teachers, union and government offcials, aid workers and institutions. Paris: UNESCO.

Randall, S. (2015). Where Have All the Nomads Gone? Fifty Years of Statistical and Demographic Invisibilities of African Mobile Pastoralists. Pastoralism, 5(1), 22. htt ps://doi.org/10.1186/s13570-015-0042-9

Randall, S. (2016). Visibilité et invisibilité statistique en Afrique. Adapter les méthodes de collecte de données aux populations ciblées. Afrique Contemporaine, 2(258), 41-57. https://doi.org/10.3917/afco.258.0041

Rhoades, A. S. (2010). Displaced Futures: Internally Displaced Persons and the Right to Education. El Rodeo: United Nations University for Peace 2010.

Sandefur, J. \& Glassman, A. (2015). The Political Economy of Bad Data: Evidence from African Survey and Administrative Statistics. Journal of Development Studies, 51(2), 116-132. https://doi.org/10.1080/00220388.2014.968138

Scheunpflug, A. \& Wenz, M. (2018). Die Globale Bildungsagenda - zu den Herausforderungen der Bildungszusammenarbeit Im Entwicklungskontext. In R. Tippelt \& B. Schmidt-Hertha (Hrsg.), Handbuch Bildungswissenschaften (S. 307-324). Berlin: Springer. https://doi.org/10.1007/978-3-531-19981-8_12
Scott, J. C. (1998). Seeing Like A State. How Certain Schemes to Improve the Human Condition Have Failed. New Haven, CT: Yale University Press.

Seitz, K. (2004). Bildung und Konflikt. Die Rolle von Bildung bei der Entstehung, Prävention und Bewältigung gesellschaftlicher Krisen - Konsequenzen für die Entwicklungszusammenarbeit. Eschborn: Deutsche Gesellschaft für Technische Zusammenarbeit.

Shanks, K. (2019). The Politics of IDP Education Provision: Negotiating Identity and Schooling in the Kurdistan Region of Iraq. International Migration, 57(2) 32-47. https://doi.org/10.1111/imig. 12545

Smith Ellison, C. \& Smith, A. (Hrsg.) (2013). Education and Internally Displaced Persons. London: Bloomsbury. https://doi.org/10.5040/9781472552914

Sommers, M. (2005). Islands of Education: Schooling, Civil War and the Southern Sudanese (1983-2004). Paris: UNESCO International Institute for Educational Planning.

Tomaševski, K. (2001). Human rights obligations: making education available, accessible, acceptable and adaptable. Right to education primers no. 3. Stockholm: SIDA.

Ullah, A., Khan, K. \& Mahmood, H. (2017). Understanding the Nexus between Conflict, Displacement, and Schooling: A Case Study of IDPs in Pakistan. Asian Journal of Peacebuilding, 5(1), 155-168. https://doi.org/10.18588/201705.00a010 UNESCO (2011). EFA Global Monitoring Report 2011. The hidden crisis: Armed conflict and education. Paris: UNESCO.

UNESCO (2018). Global Education Monitoring Report 2019: Migration, displacement and education: Building bridges, not walls. Paris: UNESCO.

UNESCO (2019). Artificial Intelligence in Education: Challenges and Opportunities for Sustainable Development. UNESCO Working Papers on Education Policy, 7. UNESCO IIEP (2010). Guidebook for planning education in emergencies and reconstruction. Paris: UNESCO International Institute for Educational Planning (IIEP).

UNESCO ILL (2020). Call for cases: Innovative literacy learning and education for refugees, migrants and internally displaced persons. Zugriff am 11.9.2020 https://uil.u nesco.org/literacy/call-cases-innovative-literacy-learning-and-education-refugeesmigrants-and-internally

UNHCR (2020). Figures at a Glance. Zugriff am 11.9.2020 https://www.unhcr.org/ figures-at-a-glance.html

UNHCR (n.d.). Binnenvertriebene. Zugriff am 19.3.2019 https://www.unhcr.org/d ach/de/ueber-uns/wem-wir-helfen/binnenvertriebene

UN OCHA (2018). World humanitarian data and trends 2018. Genf \& New York: UN Office for the Coordination of Humanitarian Affairs.

Various Organisations (2016). E-Forum Report. Planning for the Inclusion of Displaced Populations in the Education Sector. Preliminary Reflections, November 2016. UNESCO-IIEP, GPE, UNHCR, PEIC. Zugriff am 11.9.2020 https://inee.org/reso urces/planning-inclusion-displaced-populations-education-sector-preliminary-ref ections

\section{Dr. Cyril Brandt}

Bachelor of Arts Kulturwirt (Universität Duisburg-Essen, 2007-2010); Bachelor of Science Betriebswirtschaftslehre (2007-2011); Research Master International Development Studies (Universität Amsterdam, 2012-2014); $\mathrm{PhD}$ International Development Studies (Universität Amsterdam, 20142017); Postdoc (Universität Antwerpen, 2018); circa 2 Jahre Feldforschung in der Demokratischen Republik Kongo im Rahmen des Masters, PhD und Postdoc; Associate Researcher, Institute of Development Policy (Antwerp); Honorary Associate, Institute of Development Studies (Sussex); Freiberuflicher Berater und Forscher für u. a. GIZ, Cambridge Education, Save the Children, Integrity und Mokoro; Gesellschaftliche Funktionen: Planner der Special Interest Group ,Education, Conflict and Emergencies' der Comparative and International Education Society (2015-2017); Anti-Rassismus Trainer bei Phoenix e.V 